\title{
Hypertension as an atypical presentation of unilateral ureteral obstruction
}

\author{
Pedro Mantas $^{1}$ (iD), Rute Baeta Baptista ${ }^{2}$ iD, Raquel Santos ${ }^{2}$, Ana Paula Serrão ${ }^{2}$ \\ ${ }^{1}$ Department of Pediatrics, Hospital de Santarém, Santarém, Portugal \\ 2 Nephrology Unit, Hospital Dona Estefânia, Centro Hospitalar Universitário de Lisboa Central, Lisboa, Portugal
}

\section{ABSTRACT}

Ureteral obstruction (ureteropelvic or ureterovesical junction obstruction) is frequently diagnosed during the workup investigation of an asymptomatic infant or child with upper urinary tract dilatation, commonly identified in a prenatal ultrasound. In older children, recurrent lumbar pain is a red flag for ureteral obstruction. Although less frequent, hypertension may be the initial and only manifestation of ureteral obstruction. The authors present two pediatric cases of unilateral ureteral obstruction with hypertension, in which the surgical treatment of the obstruction leads to blood pressure normalisation. In all pediatric age groups, a systematic investigation for secondary causes of hypertension is of paramount importance. In some cases, especially those of an obstructive nature, early surgical management can be curative, with normalization of blood pressure levels and prevention of renal injury.

Keywords: Hypertension, ureteral obstruction, child.

(C) 2021 Portuguese Journal of Nephrology \& Hypertension. Published by Publicações Ciência \& Vida This is an open access article under the CC BY-NC-ND license (http://creativecommons.org/licenses/by-nc-nd/4.0/).

\section{INTRODUCTION}

The estimated prevalence of hypertension in the pediatric age group is $4-5 \%^{1,2}$, although it is likely underestimated due to geographical differences in hypertension definition and in the methods used for blood pressure measurement ${ }^{2}$. Portuguese data identified a higher prevalence of pediatric hypertension of approximately $13 \%^{3}$. Early detection of hypertension and its treatment are associated with better long-term cardiovascular outcomes ${ }^{1}$. There is increasing evidence that hypertension diagnosed in adulthood is often present from childhood ${ }^{1}$. In children, hypertension can be commonly diagnosed in an otherwise asymptomatic child, emphasizing the importance of screening programmes $^{1}$.

The diagnosis of hypertension in children and adolescents should always prompt the investigation for secondary causes. Hypertension in pre-adolescents most commonly has an underlying cause, whereas primary hypertension becomes predominant after the age of $10^{1,2}$. The prevalence of secondary hypertension has been reported as high as $75-85 \%$ in younger children ${ }^{2}$, mainly associated with renal parenchymal and renovascular disease $\mathrm{e}^{1,2}$. Severe hypertension, target organ damage or resistance to treatment in a pediatric patient must raise the suspicion of a secondary etiology ${ }^{2,4}$. Renal disease is responsible for up to two thirds of the cases of severe hypertension in children ${ }^{5}$.

Hydronephrosis is a common finding which can be associated with congenital anomalies, such as vesicoureteral or ureterovesical junction obstruction ${ }^{6,7}$. Most cases are detected on the antenatal ultrasounds. However, hydronephrosis may present later as intermittent lumbar or flank pain, recurrent urinary tract infections, urolithiasis and rarely hypertension $4,8,9$. The underlying mechanisms linking hydronephrosis to hypertension are still incompletely understood ${ }^{4,9}$. The published literature on this topic is scarce, although it has already been reported that as much as $10 \%$ of children with ureteral obstruction also have hypertension ${ }^{4,9-11}$. There are a few case reports of pediatric patients who became normotensive after surgical relief of the obstruction ${ }^{10,11}$.

In this manuscript, we present two pediatric cases of hypertension as the initial and only manifestation of unilateral ureteral obstruction, emphasizing the importance of a thorough investigation of secondary causes of hypertension.

\section{CASE PRESENTATION - CASE 1}

A 17-year-old male of mixed ethnicity, presenting with episodic malaise was referred to the emergency department due to newly detected stage 2 hypertension (European Society of Hypertension ${ }^{2}$ ) during an outpatient appointment. Over the past two years, he has been experiencing the bimonthly occurrence of mild bilateral frontotemporal tension headaches, recently localized over the occipital region. The headaches presented at different times during the day, did not wake him up from his sleep, and were easily controlled with paracetamol. Three weeks before his admission, he had a complaint 
of moderate colicky right flank pain, which was interpreted as muscle cramps and resolved with paracetamol.

His physical and psychomotor development were normal. His past medical history was uneventful until 14 years of age, when he had a seizure during an episode of febrile acute illness. The workup at the time excluded a central nervous system infection or structural abnormality. The electroencephalogram showed no specific abnormalities. He did not concede to tobacco or drug use and was not taking any medication at that time. He played football once a week with no fatigue. He had no recurrence of seizures.

The family history was positive for primary hypertension, diagnosed above the age of 60 , on both maternal grandparents, as well as type Il diabetes, lower-limb deep venous thrombosis and lupus erythematosus systemic on his maternal grandmother.

The initial examination revealed a blood pressure of $174 / 106 \mathrm{mmHg}$ (stage 2 hypertension according to the European Society of

\section{Figure 1}

Renal ultrasound Case 1

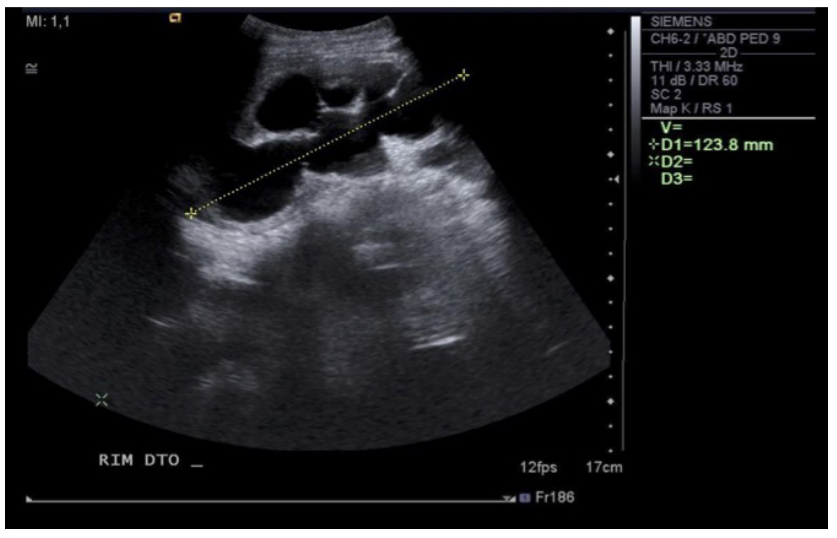

Figure 2

99mTc-MAG3 diuretic renography Case 1

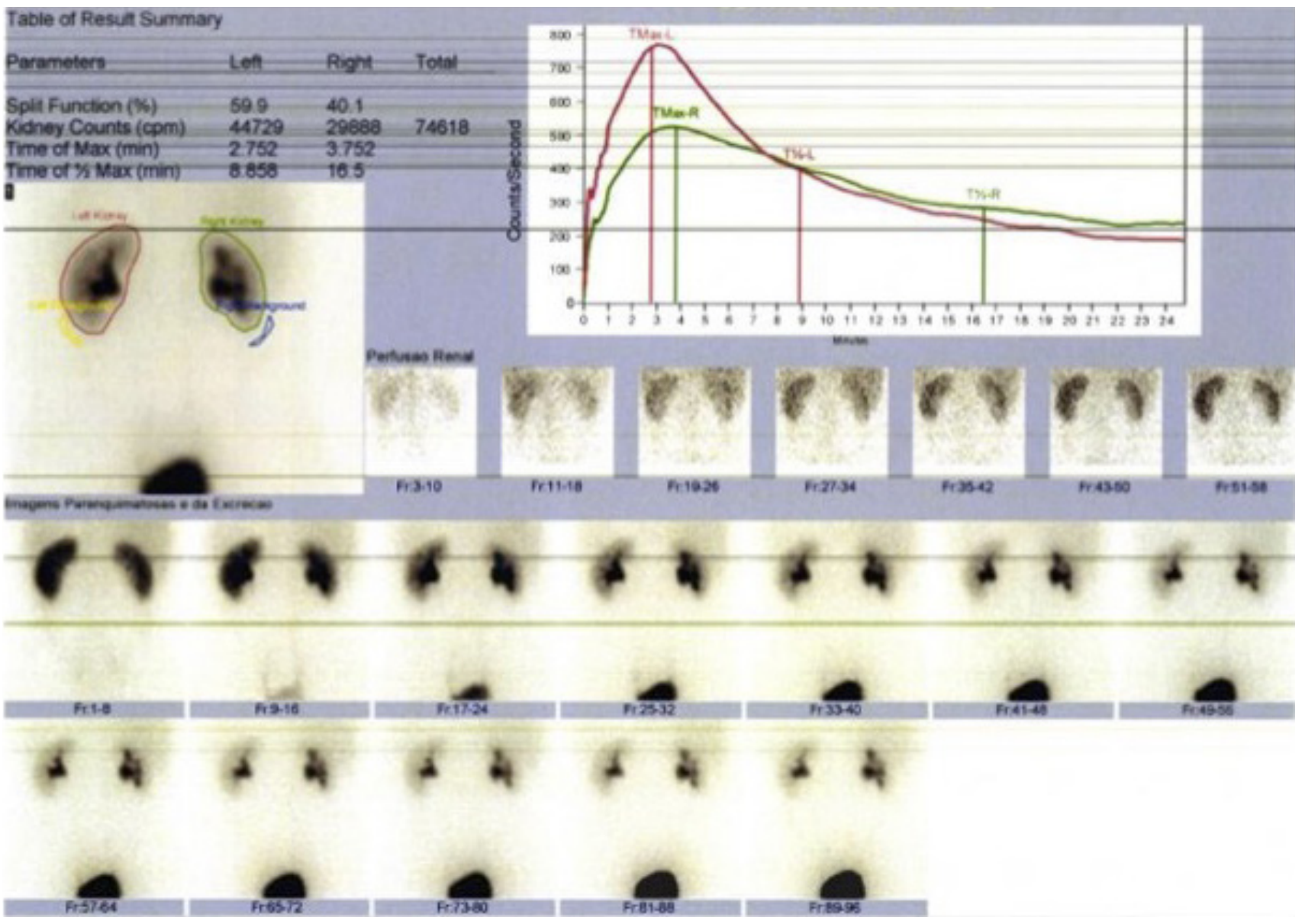


Hypertension $^{2}$ ) and a heart rate of $87 \mathrm{bpm}$. There was no significant differential between the right upper and other limbs blood pressure measurements. Radial and posterior tibial pulses were symmetrical. His body mass index was $22.2 \mathrm{Kg} / \mathrm{m}^{2}$ ( $50^{\text {th }}$ centile). He was apyrexial, with no respiratory distress or diaphoresis, and the cardiopulmonary auscultation was unremarkable. There were no palpable masses or bruits on the abdominal examination. The neurologic and ophthalmologic examinations were normal. The electrocardiography and echocardiography were normal. Blood tests revealed hemoglobin 15.3 g/dl (range 13-16), platelet count 230,000/ $\mu$ l (range 150-450), blood urea nitrogen $26 \mathrm{mg} / \mathrm{dl}$ (range 18-45), creatinine $1.44 \mathrm{mg} / \mathrm{dl}$ (range 0.66-1.1), sodium $140 \mathrm{mmol} / \mathrm{l}$ (range 136-145), potassium $3.7 \mathrm{mmol} / \mathrm{l}$ (range 3.4-4.7), chloride $101 \mathrm{mmol} / \mathrm{l}$ (range 98-107), calcium $9.7 \mathrm{mg} /$ $\mathrm{dl}$ (range 8.4-10.2), thyroid stimulating hormone $1.3 \mu \mathrm{UI} / \mathrm{ml}$ (range $0.47-3.41$ ), free thyroxine $0.9 \mathrm{ng} / \mathrm{dl}$ (range 0.89-1.37) and troponin of $2.1 \mathrm{pg} / \mathrm{ml}$ (normal<34.2). Renin and aldosterone were not screened. The estimated glomerular filtration rate calculated using the CKD-EPI equation was $82.2 \mathrm{ml} / \mathrm{min} / 1.73 \mathrm{~m}^{2}$. The toxicology screening was negative. The urinalysis showed no evidence of proteinuria, pyuria, or hematuria, urinary protein-to-creatinine ratio was $0.05 \mathrm{mg} / \mathrm{mg}$, and urinary albumin-to-creatinine ratio was $6 \mu \mathrm{g} / \mathrm{mg}$. The renal ultrasound (Figure 1) revealed right-sided hydronephrosis. The length of the right kidney was $13.3 \mathrm{~cm}$, with the renal pelvis measuring $41 \mathrm{~mm}$, dilation of the renal calyces and moderate cortical thinning despite normal echogenic parenchymal appearance with preserved corticomedullary differentiation. The long axis of the left kidney long was $12 \mathrm{~cm}$, without hydronephrosis or parenchymal abnormalities. There was no evidence of renal artery stenosis on the doppler examination. The abdominal ultrasound was otherwise unremarkable. The patient was diagnosed with stage 2 hypertension (European Society of Hypertension ${ }^{2}$ ) likely secondary to right ureteropelvic obstruction with no apparent target organ damage. He was admitted to the hospital for further investigation and management. He was started on amlodipine $0.07 \mathrm{mg} / \mathrm{kg} /$ day for blood pressure control. Evaluation of renal function by $99 \mathrm{mTc}-$ -MAG3 diuretic renography showed delayed and diminished perfusion of the right kidney leading to a renal function of $12 \%$ and $88 \%$ in the right and left kidney, respectively. Ureteral obstruction could not be confirmed nor excluded due to poor renal function. Blood pressure was progressively normalized with amlodipine, increased to $0.14 \mathrm{mg} /$ $\mathrm{kg} /$ day on day 4 of admission. A cystoscopy and retrograde pyelogram were performed on day five of admission, confirming the hydronephrosis and a right-sided ureteral stent was placed. During the procedure, a cloudy discharge was noted upon relief of the obstruction, raising the hypothesis of urolithiasis. The urinalysis showed no evidence of infection. A follow-up ultrasound revealed a reduction of the length of the right kidney to $11.6 \mathrm{~cm}$ and no evidence of hydronephrosis. The patient was discharged asymptomatic and normotensive on amlodipine, until the follow-up outpatient visits with the pediatric urologist and the pediatric nephrologist.

One month after the stent placement, blood pressure had normalized, and serum creatinine had improved to $1.40 \mathrm{mg} / \mathrm{dl}$ (estimated glomerular filtration rate $85 \mathrm{ml} / \mathrm{min} / 1.73 \mathrm{~m}^{2}$ ). The treatment with amlodipine was stopped and blood pressure was maintained within the normal range for age, sex, and height. However, the ultrasound still showed evidence of right-sided renal pelvis dilatation $(22 \mathrm{~mm})$,

\section{Figure 3}

99mTc-MAG3 diuretic renography Case 1

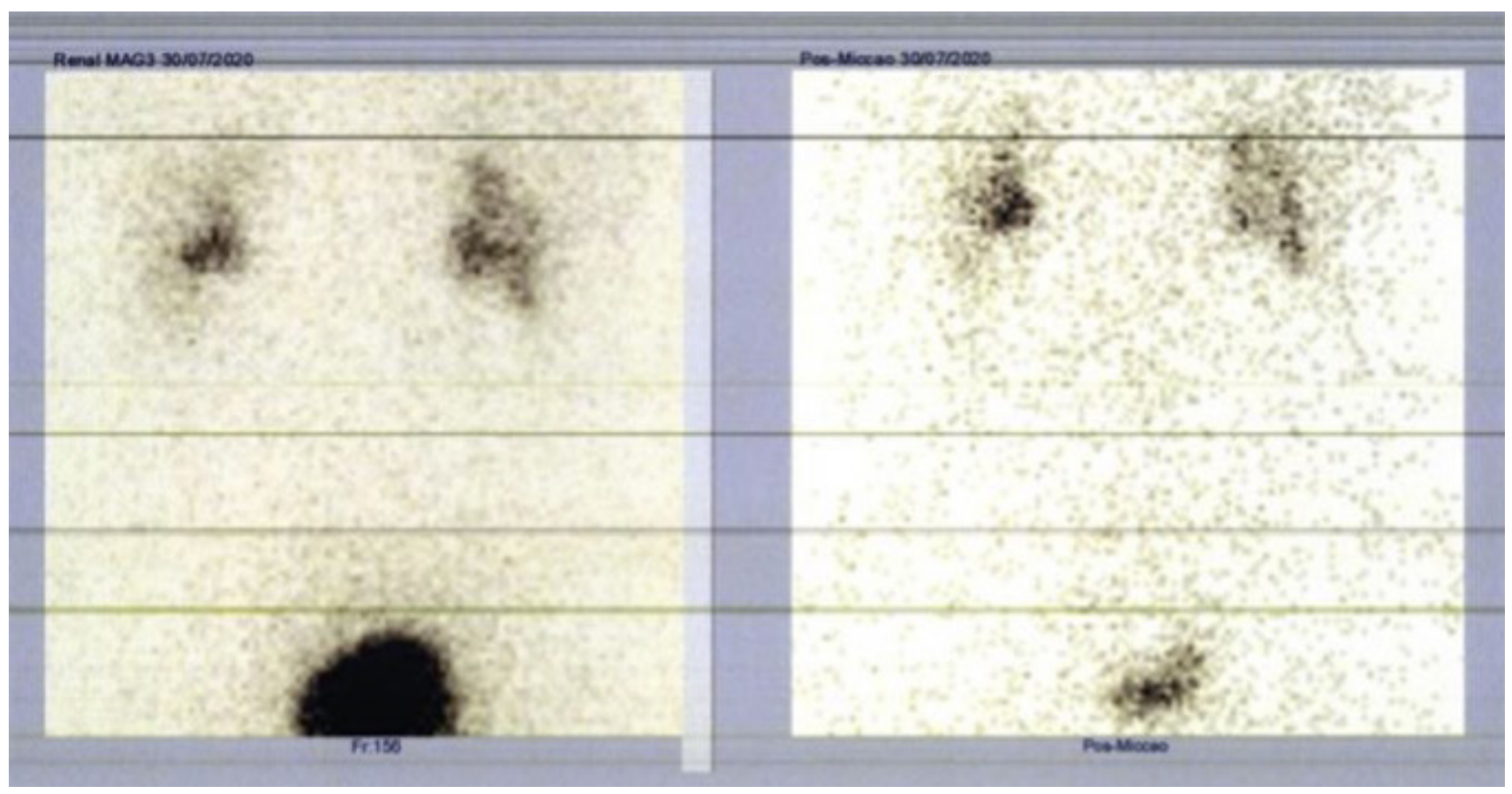


mild dilation of the renal calyces and cortical thinning, maintaining the normal echogenic appearance of the parenchyma. A computed tomography urography was performed which showed no evidence of ureteral calculi or polyps and the urinary metabolic assessment for causes of urolithiasis, including calcium, citrate, phosphorus, magnesium, uric acid, and oxalate, was unremarkable. A follow-up renal ultrasound at four months after stent placement showed complete resolution of the urinary tract dilatation and the stent was removed. One month later, five months after the initial presentation, his blood pressure was normal without any antihypertensive treatment, serum creatinine was 1.20 $\mathrm{mg} / \mathrm{dL}$ (estimated glomerular filtration rate $102.4 \mathrm{ml} / \mathrm{min} / 1.73 \mathrm{~m}^{2}$ ), there was no evidence of hydronephrosis on the renal ultrasound and the follow-up 99mTc-MAG3 diuretic renography (Figures 2 and 3) showed no obstruction and functional improvement (renal function of $41 \%$ and $59 \%$ in the right and left kidney, respectively).

\section{CASE PRESENTATION - CASE 2}

An eight-year-old Caucasian female child presented to the emergency department with brief and sudden lapses of consciousness, vomiting, sleepiness and ill-defined frontal tension headaches at the end of the day, without other symptoms or neurologic signs. At the age of two, she was diagnosed with absence seizures and treated with carbamazepine for two years. She had no recurrence of seizures and had a normal follow-up electroencephalogram. The patient had an uneventful past medical history and adequate development. She was not taking any medication at that time.

Upon physical examination the patient's blood pressure was 130/91 $\mathrm{mmHg}$ (stage 2 hypertension according to the European Society of Hypertension ${ }^{2}$ ) and her heart rate was 140 bpm, without inter-limb differences or asymmetric radial or posterior tibial pulses. She was in no respiratory distress and showed no diaphoresis. Cardiac, abdominal, and neurologic examination were normal, including ocular fundus. Her body mass index was $16.5 \mathrm{~kg} / \mathrm{m}^{2}$ (50 ${ }^{\text {th }}$ centile). Serum chemistry revealed hemoglobin $13 \mathrm{~g} / \mathrm{dl}$ (range 11.5-15.5), platelet count 218,000/ $\mathrm{ll}$ (range 180-400), blood urea nitrogen $29 \mathrm{mg} / \mathrm{dl}$ (range $15-36$ ), creatinine of $0.54 \mathrm{mg} / \mathrm{dl}$ (range $0.44-0.69$ ), sodium $140 \mathrm{mmol} / \mathrm{l}$ (range 136-145), potassium $4.7 \mathrm{mmol} / \mathrm{l}$ (range 3.4-4.7), chloride 100 $\mathrm{mmol} / \mathrm{l}$ (range 98-107) and thyroid stimulating hormone $2.29 \mu \mathrm{UI} /$ $\mathrm{ml}$ (range 0.47-3.41). Estimated glomerular filtration rate was 98.7 $\mathrm{ml} / \mathrm{min} / 1.73 \mathrm{~m}^{2}$ based on the Schwartz formula and a $k$-coefficient of 0.413 . The urinalysis showed no evidence of protein, pyuria, or hematuria. Electrocardiography was normal. Due to the patient's past medical history, she underwent electroencephalogram testing, which was unremarkable. Family history showed a maternal grandmother with early onset hypertension and history of stroke at the age of 36 .

The patient was diagnosed with stage 2 hypertension (European Society of Hypertension ${ }^{2}$ ), referred to pediatric follow-up as an outpatient and the workup for causes of secondary hypertension was performed. Ambulatory blood pressure monitoring was significant for a non-dipper, both daytime and night-time systolic (stage 2) and diastolic (stage 1) hypertension (European Society of Hypertension ${ }^{2}$ ). She had no evidence of concentric left ventricular hypertrophy on echocardiography. The renal ultrasound showed a right kidney of 12 $\mathrm{cm}$ in length, with diffuse parenchymal atrophy, absent corticomedullary differentiation and evidence of hydronephrosis with a markedly enlarged urinary tract (renal pelvis dilatation of $25 \mathrm{~mm}$, severe dilation of the renal calyces and a dilated ureter with a maximum diameter of $13 \mathrm{~mm}$ ); the left kidney measured $10 \mathrm{~cm}$ in length, without other significant changes. There was no evidence of renal artery stenosis on the Doppler examination. Additional blood workup revealed normal aldosterone and renin levels as well as normal serum and urinary catecholamines and metanephrines. The patient had no increased albuminuria. Right ureterohydronephrosis with severely compromised perfusion and function (split renal function of $40 \%$ and $60 \%$ in the right and left kidney, respectively) was revealed by $99 \mathrm{mTc}-$ -MAG3 diuretic renography, probably resulting from ureteral obstruction, unconfirmed due to poor renal function. A voiding cystourethrogram excluded vesicoureteral reflux. She was started on amlodipine $0.18 \mathrm{mg} / \mathrm{kg} /$ day for blood pressure control and referred to urology and nephrology as an outpatient.

The patient's blood pressure improved with amlodipine, although the serum creatinine worsened to $0.65 \mathrm{mg} / \mathrm{dl}$ (estimated glomerular filtration rate $81.9 \mathrm{ml} / \mathrm{min} / 1.73 \mathrm{~m}^{2}$ ). The patient underwent surgical treatment. The ureterovesical obstruction was confirmed intraoperatively, the stenotic segment was resected, a Cohen ureteric reimplantation was performed and a right-sided ureteral stent was placed. A hard light brown calculus was removed from the stenotic segment of $0.19 \mathrm{~g}$ and $7 \times 5 \times 3 \mathrm{~mm}$, testing positive for calcium and oxalate. However, the urinary metabolic assessment, including calcium, citrate, phosphorus, magnesium, uric acid, and oxalate, was unremarkable. In the follow-up ultrasound the right kidney measured $10.5 \mathrm{~cm}$ in length, maintaining diffuse parenchymal atrophy and absent corticomedullary differentiation, but showing improved hydronephrosis, with mild dilatation of the renal pelvis $(13 \mathrm{~mm})$ and moderate dilatation of the renal calyces. The stent was removed two months later. Evaluation of renal function by $99 \mathrm{mTc}-\mathrm{MAG} 3$ diuretic renography (Figures 4 and 5) nine months after the surgical intervention showed no obstruction and a split renal function of $38 \%$ and $62 \%$ in the right and left kidney, respectively. Follow-up consistently revealed normalized blood pressure measurements with antihypertensive treatment, confirmed by ambulatory blood pressure monitoring. Serum creatinine stabilized at approximately $0.65 \mathrm{mg} / \mathrm{dl}$ (estimated glomerular filtration rate $81.9 \mathrm{ml} / \mathrm{min} / 1.73 \mathrm{~m}^{2}$ ) and repeated ultrasounds had no evidence of hydronephrosis. Eight months following the surgical intervention, amlodipine was withdrawn, and the patient remained normotensive.

\section{DISCUSSION}

Ureteral obstruction is a partial or intermittent total blockage of normal urinary flow, causing hydronephrosis and progressive deterioration of renal function. It is the most common cause of antenatally detected hydronephrosis, with a reported incidence of $1 / 500$ live births ${ }^{8}$. Predominantly boys are affected and left-sided involvement is more frequent ${ }^{8}$. The etiology includes both congenital and acquired conditions, due to intrinsic narrowing or extrinsic compression of the ureter. Congenital obstruction is usually caused by stenosis of the proximal ureter, and less commonly by extrinsic compression associated with an accessory renal artery. Most cases are diagnosed during postnatal evaluation after an antenatal suspicion but may also be 


\section{Figure 4}

99mTc-MAG3 diuretic renography Case 2

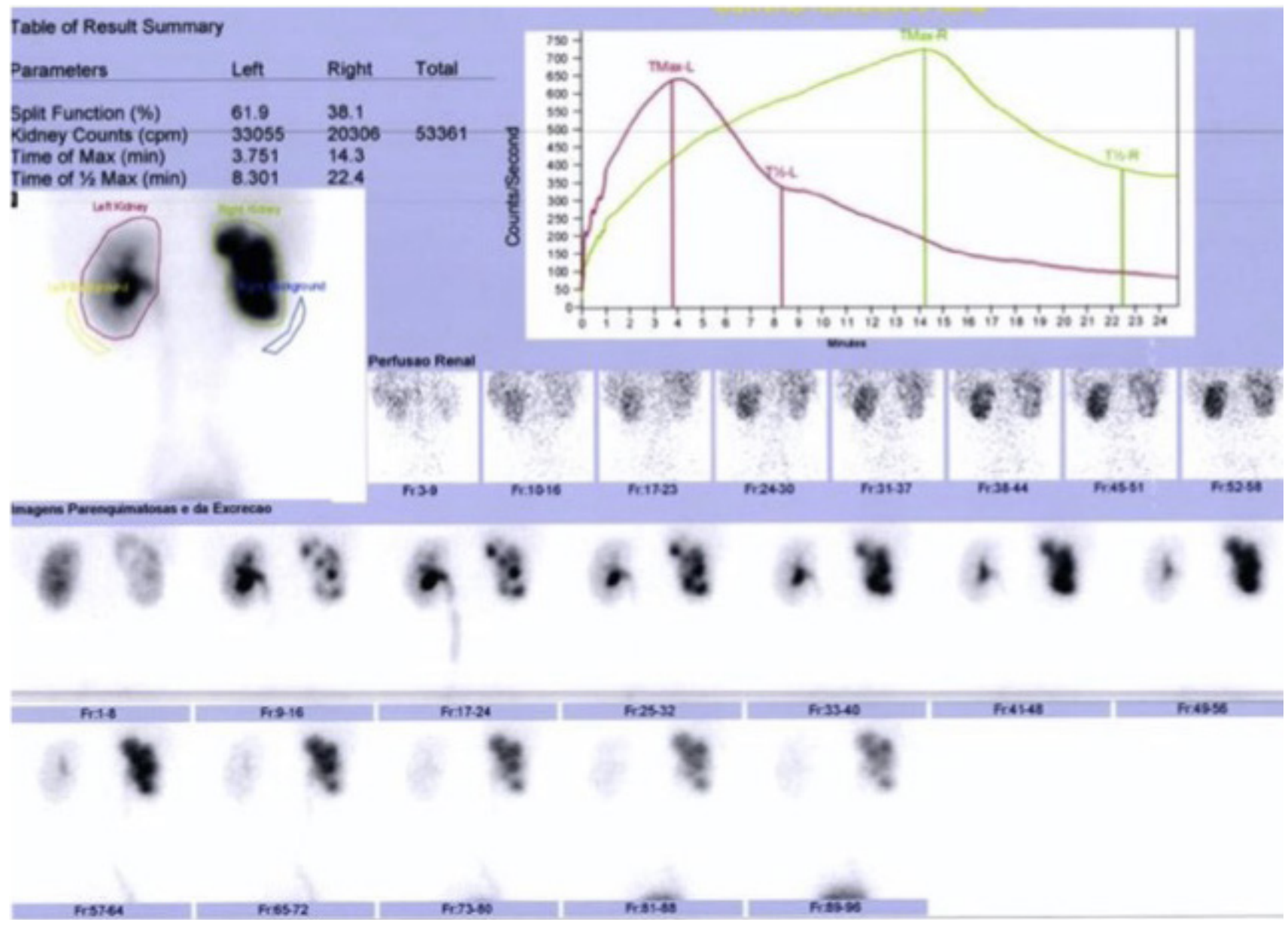

encountered in adults ${ }^{4}$. Presentation in neonates includes palpable abdominal mass due to an enlarged obstructed kidney and recurrent urinary tract infections. In older children, clinical manifestations may be intermittent lumbar or flank pain and can promote infection, urolithiasis, or hypertension. Both cases show the long-term consequences of hydronephrosis with the development of hypertension, increased risk for urolithiasis and deterioration of renal function, although renal failure as the initial presentation is unusual ${ }^{8}$. The typical clinical manifestation of flank pain was noted on the anamnesis revision of patient 1 , and patient 2 presented with symptomatic hypertension. In our cases, as is frequent in these cases, suspicion of ureteral obstruction arose from the incidental finding of hydronephrosis on imaging studies, typically ultrasound, and was confirmed by diuretic renography ${ }^{8}$. In the differential diagnosis, vesicoureteral reflux should always be screened.

Hypertension, as a complication of unilateral hydronephrosis, is still only somewhat understood. Ureteral obstruction activates vasoactive and immunological factors that lead to altered glomerular hemodynamic and tubular function, resulting in tubular atrophy, interstitial inflammation and subsequently loss of nephrons ${ }^{9}$. Mechanisms involved in this hydronephrosis-induced hypertension seem to be increased activity of the renin-angiotensin-aldosterone system, oxidative stress, nitric oxide deficiency and renal sympathetic nerve activity $^{4,9}$. Thus, unilateral hydronephrosis appears sufficient to cause hypertension, regardless of a normal contralateral kidney. Animal models suggest that the developed hypertension is salt-sensitive and directly correlated to the degree of obstruction ${ }^{9}$. Relief of this obstruction attenuates both hypertension and salt-sensitivity, whereas removal of the non-obstructed kidney enhances these variables, suggesting that the hypertensive mechanisms are primarily located within the diseased kidney and reversible ${ }^{9}$. Animal and human studies have shown that repair in the setting of obstruction and/or removal of a poorly functioning kidney can improve hypertension 4 .

Management of symptomatic children with evidence of hydronephrosis on imaging exams usually requires surgical intervention ${ }^{8}$. Renal function may be preserved or improved with the correction of the 


\section{Figure 5}

99mTc-MAG3 diuretic renography Case 2

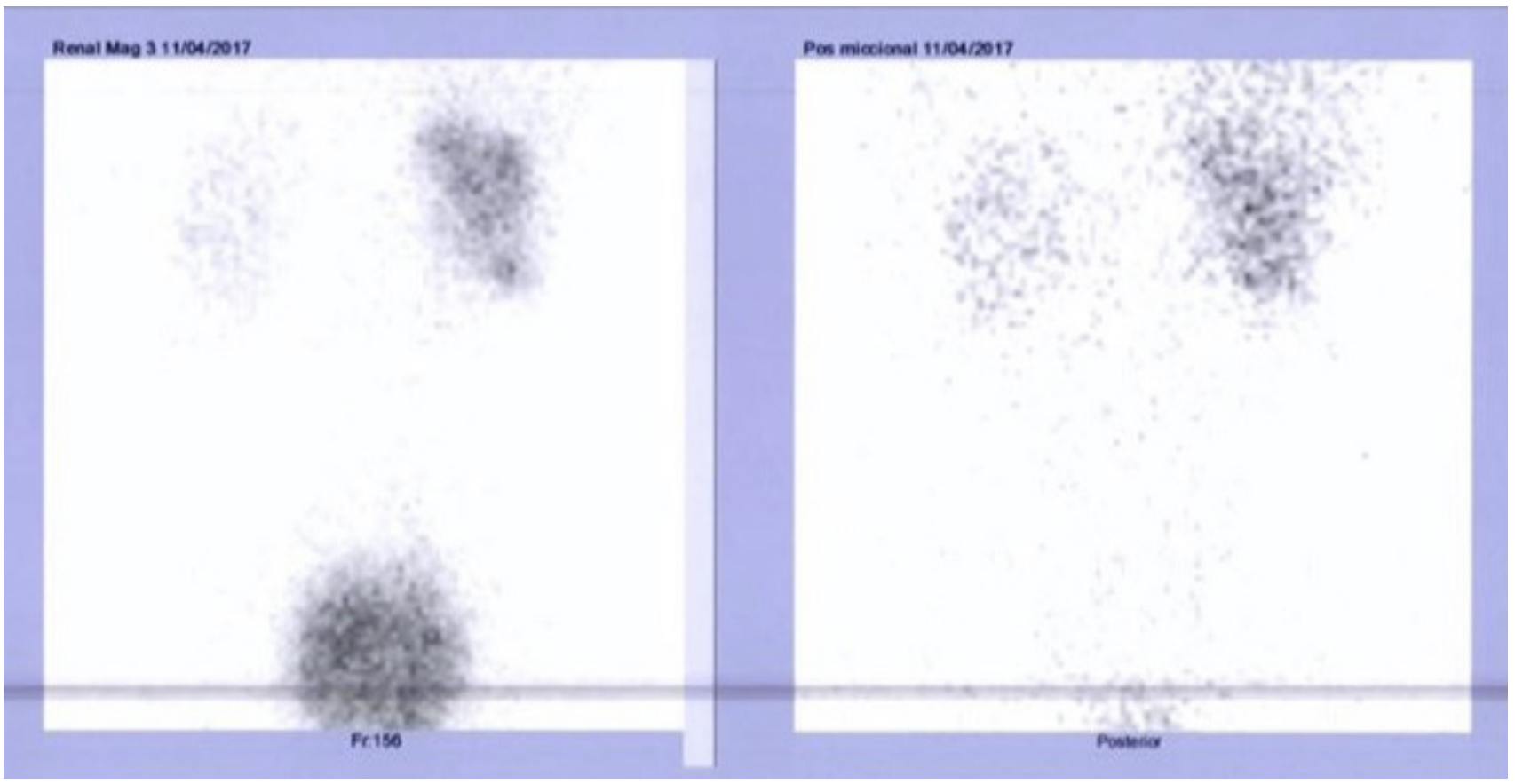

blockage and long-term complications can be prevented. Failure rate of surgical repair is low ${ }^{8}$. In asymptomatic patients, a conservative approach is often preferred, by watchful observation and surgical intervention only when there is a deterioration in renal function, development of symptoms, increase in or severe hydronephrosis 4,8 . The natural course of ureteral obstruction is variable; however most patients will show stable renal function and the hydronephrosis may improve spontaneously over time ${ }^{8}$. Follow-up with repeated ultrasound examinations is recommended every 12 to 18 months or more frequently when hydronephrosis is more severe ${ }^{8}$. Diuretic renography is warranted if there is worsening of hydronephrosis ${ }^{8}$. Postoperatively, renal ultrasonography is performed after 4 to 6 weeks, and then at increasing time intervals if decreasing severity of hydronephrosis is noted $^{8}$. Absence of improvement warrants a diuretic renography ${ }^{8}$. The proportion of children with conservative approach that become symptomatic and/or require surgical intervention is unknown. Likewise, the difference in long-term renal function between a conservative versus surgical approach remains uncertain.

Currently, clinical studies about the long-term effects on blood pressure following conservative or surgical management are lacking. A retrospective study conducted by de Waard et al. concluded that approximately $5 \%$ of children with ureteral obstruction were hypertensive and relief of the obstruction normalized blood pressure in more than $90 \%$ of all cases ${ }^{11}$. Published case reports in adults and studies with larger adult populations are few, but support resolution of hypertension following the removal of the affected kidney or relief of the obstruction ${ }^{4,10}$. In an ongoing retrospective cohort study, blood pressure was significantly reduced following relief of the obstruction in 48 adult patients that underwent surgical management of hydronephrosis due to ureteral obstruction ${ }^{9}$. Two prospective clinical studies including pediatric subjects showed that ambulatory blood pressure in hydronephrotic patients due to ureteral obstruction was significantly higher before surgery compared with that measured 6 months following surgical management ${ }^{12,13}$. Furthermore, hydronephrotic patients had higher blood pressure compared with age-matched and healthy children ${ }^{13}$. Renal function evaluated by diuretic renography suggested a positive correlation between the degree of reduced function in the affected kidney and the reduction of blood pressure following surgery ${ }^{12}$. Oxidative stress markers with abnormal levels were also reduced 6 months post-correction of the ureteral obstruction ${ }^{12}$. Case reports and studies in both adult and pediatric populations indicate that recurrence of obstruction following surgical management was associated with hypertension and that blood pressure was again reduced after relief of the obstruction ${ }^{4,10}$. In our cases, blood pressure was initially managed with oral antihypertensive medication. The surgical relief of the obstruction lead to blood pressure normalisation even after antihypertensive medication withdrawal. Additionally, in case 1, postoperative serum creatinine levels and renogram suggested improved renal function. In case 2 , on the other hand, despite some improvement in serum creatinine, the obstruction resulted in irreversible renal dysfunction as revealed by the follow-up renography.

The cases presented of ureteral obstruction diagnosed during the workup for hypertension emphasize the importance of a thorough investigation. In both cases, long-standing ureteral obstruction resulted 
in significant hydronephrosis with severely compromised renal function. Early surgical management of ureteral obstruction seems to be potentially curative leading to blood pressure normalisation and renal function improvement.

Disclosure of potential conflicts of interest: none declared.

\section{References}

1. Mattoo TK. Epidemiology, risk factors, and etiology of hypertension in children and adolescents. In: UpToDate, Post, TW (Ed), UpToDate, Waltham, MA, 2020.

2. Lurbe E, Agabiti-Rosei E, Cruickshank JK, et al. 2016 European Society of Hypertension guidelines for the management of high blood pressure in children and adolescents. J Hypertens 2016 Oct;34(10):1887-1920

3. Maldonado J, Pereira T, Fernandes R, et al. An approach of hypertension prevalence in a sample of 5381 Portuguese children and adolescents. The AVELEIRA registry. "Hypertension in Children." Blood Press 2011 Jun;20(3):153-157.

4. Toocheck C, Guerrero T. Tragedy of transition: hypertensive crisis in a young adult secondary to unilateral ureteropelvic junction obstruction following pyeloplasty as an adolescent. BMJ Case Rep 2018 Aug 29;2018:bcr2018225815.

5. Kay JD, Sinaiko AR, Daniels SR. Pediatric hypertension. Am Heart J 2001 Sep;142(3):422-432.

6. Baskin LS. Overview of fetal hydronephrosis. In: UpToDate, Post, TW (Ed), UpToDate, Waltham, MA, 2020.

7. Baskin LS. Postnatal management of fetal hydronephrosis. In: UpToDate, Post, TW (Ed), UpToDate, Waltham, MA, 2020.
8. Baskin LS. Congenital ureteropelvic junction obstruction. In: UpToDate, Post, TW (Ed), UpToDate, Waltham, MA, 2020.

9. Carlström M. Hydronephrosis and risk of later development of hypertension. Acta Paediatr 2019 Jan;108(1):50-57.

10. Al-Mashhadi A, Häggman M, Läckgren G, et al. Changes of arterial pressure following relief of obstruction in adults with hydronephrosis. Ups J Med Sci 2018 Dec;123(4):216-224.

11. de Waard D, Dik P, Lilien MR, et al. Hypertension is an indication for surgery in children with ureteropelvic junction obstruction. J Urol 2008 May;179(5):1976-1979.

12. Al-Mashhadi A, Checa A, Wahlin N, et al. Changes in arterial pressure and markers of nitric oxide homeostasis and oxidative stress following surgical correction of hydronephrosis in children. Pediatr Nephrol 2018 Apr;33(4):639-649.

13. Al-Mashhadi A, Neveus T, Stenberg A, et al. Surgical treatment reduces blood pressure in children with unilateral congenital hydronephrosis. J Pediatr Urol 2015 Apr;11(2):91.e1-e6.

\section{ORCID}

Pedro Mantas (iD) 0000-0003-1741-3023

Rute Baeta Baptista (iD) 0000-0002-7572-2812

\section{Correspondence to:}

Pedro Mantas

Hospital de Santarém

Av. Bernardo Santareno 3737B, 2005-177 Santarém

E-mail: pedromantas@gmail.com 